\title{
Mathematical Modeling of the 2019 Measles Outbreak on US Population
}

\author{
Durgesh N Sinha $^{1 *}$, Nicholas Klahn ${ }^{2}$ and Samiye Pehlivan ${ }^{3}$ \\ ${ }^{1}$ Adjunct Assistant Professor, Temple University, Philadelphia, Strayer University, Row- \\ an College at Burlington County, Community College of Philadelphia, Mercer County \\ Community College, New Jersey, USA \\ ${ }^{2}$ Senior student of Medical track, Rowan College at Burlington County, New Jersey, USA \\ ${ }^{3}$ Sophomore student of Medical track, Rowan College at Burlington County, New Jersey, \\ USA \\ *Corresponding Author: Durgesh N Sinha, Adjunct Assistant Professor, Temple Univer- \\ sity, Philadelphia, Strayer University, Rowan College at Burlington County, Community \\ College of Philadelphia, Mercer County Community College, New Jersey, USA.
}

\section{Abstract}

This paper analyzes the 2019 measles epidemic in the United States through a developed mathematical model. We simulated our model in MATLAB using differential equations and found that in vaccinated populations, viral spread was severely hindered when compared to populations that were unvaccinated. The lack of viral prevalence was backed up by the basic reproduction number being less than one $(\mathrm{R} 0=0.03976)$.

Keywords: Mathematical Modeling; Measles; MATLAB

\section{Introduction}

Measles is a highly infectious virus that results in fever, rash, cough, conjunctivitis, and in about four days after symptom onset, rashes all over the skin and in the mouth [1]. More severe symptoms that can result in death are pneumonia and encephalitis, however, both are rare with only $6 \%$ of cases and $0.1 \%$ of cases of each complication occurring per measles case [1,2]. Another complication that lasts after the infection is immunosuppression [3] The diminished immune system leaves a person more susceptible to opportunistic infection. But once the person recovers, they will not be able to get measles again [4].

The symptomatic period results in a viral spread occurring over an eight-day period [2]. Measles is spread by droplet nuclei, meaning that the virus is able to spread through the air which is the main contributor to its high infectivity [2]. Due to the disease being so infectious, those that have it should be isolated from others in order to prevent widespread viral spreading [1].

Fortunately, there is a vaccine in order to prevent the spread of the infection within a population. The measles-mumps-rubella (MMR) vaccine is a two-dose vaccine [3]. This means that a person must get both doses in order to receive the full benefits of the vaccine. That being said, a single dose is still effective for preventing measles. According to a study by Giuseppe La Torre, those that were unvaccinated had $0.4 \%$ of the participants end up contracting measles, whereas only $0.06 \%$ of the participants in the single

\section{Received: March 11, 2020}

Published: March 31, 2020

(C) All rights are reserved by Durgesh N Sinha., et al. dose group had gotten measles [3]. Those that got both doses had no cases of measles [3]. The vaccine is usually given in childhood, and although measles is thought of as childhood disease, over half of the cases occur in adults $[2,3]$.

From this information, we developed a model to analyze the 2019 United State measles epidemic. This epidemic was the worst outbreak of measles in 27 years with a majority of the cases (89\%) occurring in those that were unvaccinated [5]. There was a broad age range of those infected with most being in children of various ages, but 29\% were adults [5]. Although there were some reports of more serious complications, no deaths had been reported $[5,6]$.

Derivation of model formation

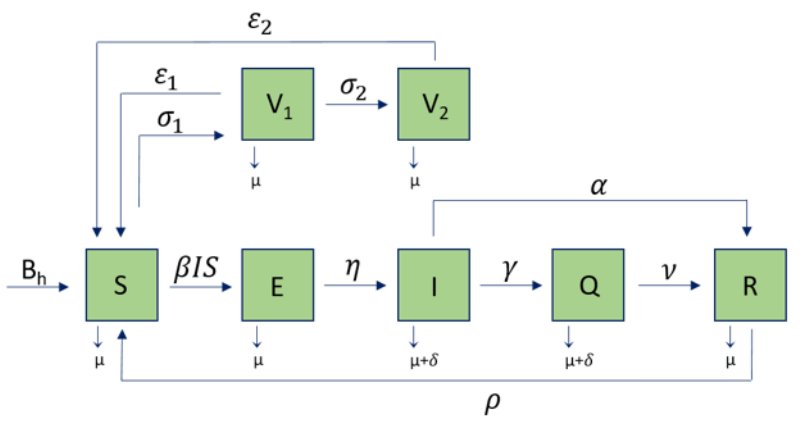

Figure 1: The measles model. 


\section{Description of model parameters}

\begin{tabular}{|c|c|}
\hline$S(t)$ : Susceptible human in timet & $E(t)$ : Exposed human in timet \\
\hline $\mid(t):$ Infectious human in timet & $Q(t):$ Quarantined human in timet \\
\hline$R(t):$ Recovered humans in time $t$ & $V_{1}(t): 1^{s t}$ dose vaccinated humans in timet \\
\hline$V_{2}(t): 2^{\text {nd }}$ dose vaccinated humans in timet & $\mathrm{N}_{h}(t)$ : Total human population in timet \\
\hline$B:$ Birth rate of humans & $\beta:$ Infectivity of measles \\
\hline$\eta$ : Rate of transmission from exposed to infected human & $v:$ Rate of transmission from quarantined to recovered human \\
\hline$\alpha$ : Rate of transmission from infected to recovered humans & $\rho:$ Rate of transmission from recovered to susceptible humans \\
\hline $\begin{array}{l}\sigma_{1} \text { : Rate of transmission from susceptible to 1st dose vaccinated } \\
\text { human in timet }\end{array}$ & $\begin{array}{l}\sigma_{2} \text { : Rate of transmission of } 1^{\text {st }} \text { dose vaccinated to } 2^{\text {nd }} \text { dose } \\
\text { vaccinated human in timet }\end{array}$ \\
\hline $\begin{array}{l}\varepsilon_{1}: \text { Rate of transmission from } 1^{\text {st }} \text { dose vaccinated to susceptible } \\
\text { human in timet }\end{array}$ & $\begin{array}{l}\varepsilon_{2} \text { : Rate of transmission of } 2^{\text {nd }} \text { dose vaccinated to susceptible human } \\
\text { in timet }\end{array}$ \\
\hline H Natural death & Death rate of humans due to measles \\
\hline
\end{tabular}

Table 1: Description of the parameters within the measles model.

\section{Model formation}

The model that we made is a basic SEIR model with the addition of a hospitalized class $(Q)$ and two vaccination classes to represent each vaccine dose with $V_{1}$ being the first dose, and $V_{2}$ being the second. Our model starts in the susceptible group (S), these people are able to be infected with measles (through the infectivity rate of $\beta$ ), or they can receive their first vaccine dose $\left(\sigma_{1}\right)$. Those that get the first vaccine dose can become infected $\left(\varepsilon_{1}\right)$ or recieve the second dose $\left(\sigma_{2}\right)$. Those in $V_{2}$ will stay there unless they die or they become susceptible to infection $\left(\varepsilon_{2}\right)$. On the disease side of the model, after entering the exposed class (E), they will have to wait for incubation period of measles to pass $(\eta)$, before they become infectious. Once infectious (I), they can either recover at home with no medical intervention $(\alpha)$ or go to the hospital $(\gamma)$ and recover there $(v)$. Measles can be fatal so the infectious groups both have an additional death parameter other than natural death $(\mu)$, as death due to measles $(\delta)$.

Equations forms based on derived model

$$
\begin{aligned}
& \frac{d S}{d t}=\beta_{h} N_{h}-\beta I S+\varepsilon_{1} V_{1}+\varepsilon_{2} V_{2}-\mu S+\rho R \\
& \frac{d E}{d t}=\beta I S-\eta E-\mu E \\
& =\beta I S-(\eta+\mu) E \\
& \frac{d I}{d t}=\eta E-\alpha I-\gamma I-(\mu+\delta) I \\
& =\eta E-(\alpha+\gamma+\mu+\delta) I \\
& \frac{d Q}{d t}=\gamma I-v Q-(\mu+\delta) Q \\
& =\gamma I-(v+\mu+\delta) Q \\
& \frac{d R}{d t}=v Q-\mu R+\alpha I-\rho R \\
& =v Q+\alpha I-(\mu+\rho) R \\
& \frac{d V_{1}}{d t}=\sigma_{1} S-\sigma_{2} V_{1}-\varepsilon_{1} V_{1}-\mu V_{1} \\
& =\sigma_{1} S-\left(\sigma_{2}+\varepsilon_{1}+\mu\right) V_{1} \\
& \frac{d V_{2}}{d t}=\sigma_{2} V_{1}-\varepsilon_{2} V_{2}-\mu V_{2} \\
& =\sigma_{2} V_{1}-\left(\varepsilon_{2}+\mu\right) V_{2} \\
& N_{h}(t)=S(t)+V_{1}(t)+V_{2}(t)+\varepsilon_{t}+I(t)+Q(t)+R(t)
\end{aligned}
$$

\section{Stability of the model}

We were able to prove that the model is globally and locally stable for the disease-free equilibrium. All of our parameters are positive or non-negative therefore all parameters must remain positive or non-negative for positive initial conditions for $t \geq 0$. From the model equations we have:

$$
\frac{d N_{h}}{d t}=\beta_{h}-\mu N_{h}-S I-S Q \leq \beta_{h}-\mu_{h} N_{h}
$$

The closed set

$$
\begin{aligned}
& \mathrm{D}=\left\{\left(S, E, I, Q, R, V_{1}, V_{2}\right) \in R_{+}\right\} \\
& N_{h} \leq \frac{\beta_{h}}{\mu}
\end{aligned}
$$

is a feasible region for the graphs.

\section{Basic reproduction number}

The basic reproduction number is the amount of secondary infectious cases caused by a single infectious case within a susceptible population. The basic reproductive equations are as follows:

$$
\begin{aligned}
& R_{0}^{h}=\frac{1}{S}=\frac{\eta \beta}{(\eta+\mu)(\alpha+\gamma+\mu+\delta)} \\
& R_{0}^{h} \text { (value) }=0.03976
\end{aligned}
$$

Qualitative analysis Disease-free equilibrium

Theorem 1: The system (1) asymptotically stable for disease-free equilibrium, when:

$R_{0}^{h}<1$

Proof: Jacobian matrix of the system is as follows:

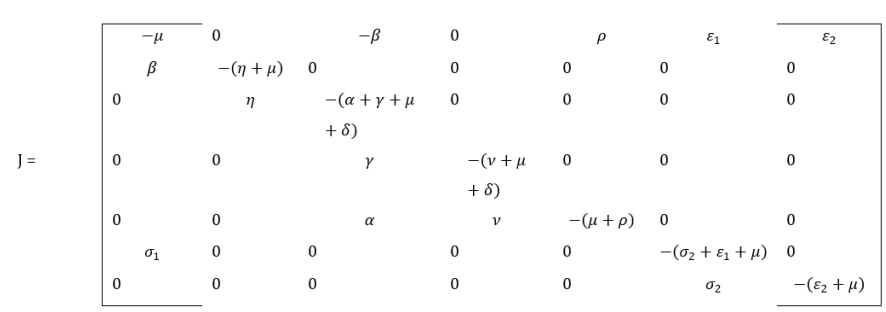

Due to large matrix, we have considered the block matrix. The diagonals for the Jacobian matrix of system 1 :

$$
\begin{aligned}
& A_{1}=\left|\begin{array}{c}
-\mu 0 \\
\beta-(\eta+\mu)
\end{array}\right| \\
& A_{2}=\left|\begin{array}{c}
-(\alpha+\gamma+\mu+\delta) 0 \\
\gamma-(\gamma+\mu+\delta)
\end{array}\right| \\
& A_{3}=\left|\begin{array}{c}
-(\mu+\rho) 0 \\
0-\left(\sigma_{2}+\varepsilon_{1}+\mu\right)
\end{array}\right| \\
& A_{4}=-\left|\varepsilon_{2}+\mu\right|
\end{aligned}
$$

For calculation of $\Delta A_{1}$ :

$$
\begin{aligned}
& \text { Trace }\left(A_{1}\right)=-(\mu+\varphi+\mu) \\
& \operatorname{det}\left(A_{1}\right)=\mu(\eta+\mu) \\
& \Delta A_{1}(\mathrm{t})=\mathrm{t}^{2}+(\mu+\eta+\mu) \mathrm{t}+\mu(\eta+\mu) \\
& =(t+\mu)(t+\eta+\mu)
\end{aligned}
$$


For calculation of $\Delta A_{2}$

Trace $\left(A_{2}\right)=-(\alpha+\gamma+\mu+\delta+v+\mu+\delta)$

$\operatorname{det}\left(A_{2}\right)=(\alpha+\gamma+\mu+\delta)(v+\mu+\delta)$

$\Delta A_{2}(\mathrm{t})=\mathrm{t}^{2}+(\alpha+\gamma+\mu+\delta+v+\mu+\delta) \mathrm{t}+(\alpha+\gamma+\mu+\delta)(v+\mu+\delta)$

$=(t+\alpha+\gamma+\mu+\delta)(t+v+\mu+\delta)$

For calculation of $\Delta A_{3}$

Trace $\left(A_{3}\right)=-\left(\mu+\rho+\sigma_{2}+\varepsilon_{1}+\mu\right)$

$\operatorname{det}\left(A_{3}\right)=(\mu+\rho)\left(\sigma_{2}+\varepsilon_{1}+\mu\right)$

$\Delta A_{3}(\mathrm{t})=t^{2}+\left(\mu+\rho+\sigma_{2}+\varepsilon_{1}+\mu\right) \mathrm{t}+(\mu+\rho)\left(\sigma_{2}+\varepsilon_{1}+\mu\right)$

$=(t+\mu+\rho)\left(t+\sigma_{2}+\varepsilon_{1}+\mu\right)$

$\Delta A_{4}(\mathrm{t})=t+\left(\varepsilon_{2}+\mu\right)$

and for the full Jacobian

$\Delta A(\mathrm{t})=\Delta A_{1}(\mathrm{t}) \cdot \Delta A_{2}(\mathrm{t}) \cdot \Delta A_{3}(\mathrm{t}) \cdot \Delta A_{4}(\mathrm{t})$

$=(t+\mu)(t+\eta+\mu)(t+\alpha+\gamma+\mu+\delta)(t+v+\mu+\delta)(t+\mu+\rho)$

$\left(t+\sigma_{2}+\varepsilon_{1}+\mu\right)\left(t+\varepsilon_{2}+\mu\right)$

The eigen values are:

$\lambda_{1}=-\mu$

$\lambda_{2}=-(\eta+\mu)$

$\lambda_{3}=-(\alpha+\gamma+\mu+\delta)$

$\lambda_{4}=-(v+\mu+\delta)$

$\lambda_{5}=-(\mu+\rho)$

$\lambda_{6}=-\left(\sigma_{2}+\varepsilon_{1}+\mu\right)$

$\lambda_{7}=-\left(\varepsilon_{2}+\mu\right)$

Hence all eigen values $\lambda_{1}, \lambda_{2}, \lambda_{3}, \lambda_{4}, \lambda_{5}, \lambda_{6}, \lambda_{7}$ are negative, when $R_{0}^{h}<1$. This proves that our system of human population is locally asymptotically stable when $R_{0}^{h}<1$.

Global stability of disease-free equilibrium

We show the global stability of the model given by kamkang and Sallet [12]. In this method, to show global stability, the model has to satisfy the five hypotheses, which has been summarized briefly in Appendix.

Theorem 2: States that the system (1) in globally stable for disease free equilibrium when $\mathrm{R}_{0}{ }^{\mathrm{h}}<1$.

Proof: We have shown above that:

$\mathrm{D}=\left\{\left(S, E, I, Q, R, V_{1}, V_{2}\right) \in R_{+}^{7}: N_{H} \leq \frac{\beta_{h}}{\mu}\right\}$ is bounded and positively in variant in $\mathrm{R}_{+}^{7}$, where the hypothesis $\mathrm{A}_{1}$, and $\mathrm{A}_{2}$ are satisfied.

In our model

$x_{1}=\left(S, Q, R, V_{1}, V_{2}\right)$

$x_{2}=(E, I)$

$x=\left(x_{1}, x_{2}\right)$

The matrix $A_{2}(x)$ is given by

$A_{2}(x)=\left[\begin{array}{c}-(\eta+\mu) \beta S \\ \eta-(\alpha+\gamma+\mu+\delta\end{array}\right]$
As requested by hypothesis $\mathrm{A}_{3}$, for any $\quad \chi \in R_{+}^{7}$, natrix is irreducible. Now for hypothesis $A_{4}$, there is a maximum and uniquely realized in $R_{+}{ }^{7}$ if $S=1$ at DFE. The maximum matrix $J_{2}$, the block of Jacobian at DFE corresponding to the matrix $A_{2}(x)$ is given by

$J_{2}=\left[\begin{array}{c}-(\eta+\mu) \beta \\ \eta-(\alpha+\gamma+\mu+\delta\end{array}\right]$

For hypothesis $A_{4}$, the diogonal matrix $A_{11}{ }^{2}$ is bounded by the matrices

$A_{11}^{-2}=\left[\begin{array}{c}-(\eta+\mu) \beta \\ \eta-(\alpha+\gamma+\mu+\delta\end{array}\right]$

Which is the maximum. The maximum is realized at each point at manifold $\mu(E=0, I=0)$. This implies that these points belong to the manifold with equation $\mathrm{E}=\mathrm{I}=0$. Thus, hypothesis $\mathrm{A}_{4}$ is satisfied.

Now for hypothesis $A_{5}$, the condition $\alpha\left(A_{11}{ }^{2}\right) \leq 1$. Thus hypothesis $A_{5}$ is equivalent to $R_{0}{ }^{h}<1$. This proves that the model is globally stable for disease- free equlibrium when $\mathrm{R}_{0}{ }^{\mathrm{h}}<1$.

\section{Endemic equilibrium}

Besides the disease-free equilibrium point we shall show that the formulated model (1) has an epidemic equilibrium point. The endemic equilibrium point is a positive steady state solution where the disease persists in the population. Solving the system of equations (1) at endemic steady state resulted in

$$
P^{*}=\left(S^{*}, E^{*}, I^{*}, Q^{*}, R^{*}, V_{1}^{*}, V_{2}^{*}\right)
$$$$
E^{*}>0, I^{*}>0, Q^{*}>0
$$

Theorem 3: The unique boundary equilibrium of the model equations (1) is globally asymptotically stable in (1) whenever $\mathrm{R}_{0}{ }^{\mathrm{h}}>1$.

Proof: Considering the model equations (1) and $R_{0}{ }^{h}>1$, so that the associated unique endemic equlibrium $\mathrm{P}^{*}$ of the model exists. We consider the following non-linear Lyapunov function of Cohn Volterra tvpe:

$Z_{i}=\sum_{i=1}^{n} C_{i}\left(\chi_{i}-\chi_{i}^{*}-\chi_{i}^{*} \ln \frac{\chi_{i}}{\chi_{i}^{*}}\right.$

Where $\chi$ is the variables and $\mathrm{C}_{\mathrm{i}}$ are constants. This criterion has been used in establishing the stability or otherwise of many diseases' models and also present in [13-15].

$$
\begin{aligned}
& \text { Let } S>S^{*}, E>E^{*}, I>I^{*}, Q>Q^{*}, R>R^{*}, V_{1}>V_{1}^{*}, V_{2}>V_{2}^{*} \\
& Z_{1}=S-S^{*}-S^{*} \operatorname{Ln} \frac{S}{S^{*}} \\
& Z_{1}^{1}=-\left(\frac{S^{*}-S}{S}\right)\left[B_{h} N_{h}-\beta I S+\varepsilon_{1} V_{1}+\epsilon_{2} V_{2}-\mu S+\alpha R\right]
\end{aligned}
$$

For the equilibrium relation:

$B_{h} N_{h}=\beta I^{*} S^{*}-\varepsilon_{1} V_{1}-\varepsilon_{2} V_{2}+\mu S^{*}-\alpha R$ 
$Z_{1}^{1}=-\left(\frac{S^{*}-S}{S}\right)\left[\beta I^{*} S^{*}-\varepsilon V_{1}-\varepsilon V_{2}+\mu S^{*}-\alpha R-\beta I S+\varepsilon_{1} V_{1}+\varepsilon_{2} V_{2}-\mu S+\alpha R\right]$

$Z_{1}^{1}=-\left(\frac{S^{*}-S}{S}\right)\left[\beta I^{*} S^{*}+\beta I S+\mu S^{*}-\mu S\right]$

$Z_{1}^{1}=-\left(\frac{S^{*}-S}{S}\right)\left[\beta\left(I^{*} S^{*}-I S+\mu\left(S^{*}-S\right]<0\right.\right.$

So $Z_{1}^{1} \leq 0$

$Z_{1}=E-E^{*}-E^{*} \operatorname{Ln} \frac{E}{E^{*}}$

$Z_{1}^{1}=-\left(\frac{E^{*}-E}{E}\right)[\beta I S-(\eta+\mu) E]$

For the equilibrium relation:

$Z_{2}^{1} \leq-\left(E^{*}-E\right)\left[\frac{\beta I^{*} S^{*}}{E^{*}}-(\eta+\mu)\right]$

Now form study of equation 1 at equilibrium equation.

$\beta I^{*} S^{*}=(\eta+\mu) E^{*}$

$\frac{S^{*}}{E^{*}}=\frac{(\eta+\mu)}{\beta I^{*}}$

So $Z_{2}^{1}=-\left(E^{*}-E\right)\left[\frac{\beta I^{*}(\eta+\mu)}{\beta I^{*}}-\left(\eta+\mu_{H}\right]\right.$

$Z_{2}^{1} \leq 0$

$Z_{3}^{1}=-\left(\frac{I^{*}-I}{I}\right)[\eta E-(\alpha+\gamma+\mu+\delta) I]$

For equilibrium

$\eta E^{*}=(\alpha+\gamma+\mu+\delta) I^{*}$

$\frac{E^{*}}{I^{*}}=\frac{(\alpha+\gamma+\mu+\delta)}{\eta}$

$Z_{3}^{1} \leq-\left(I^{*}-I\right)\left[\frac{\eta E^{*}}{I^{*}}-(\alpha+\gamma+\mu+\delta)\right]$

$Z_{3}^{1} \leq-\left(I^{*}-I\right)\left[\eta \frac{(\alpha+\gamma+\mu+\delta)]}{\eta}-(\alpha+\gamma+\mu+\delta)\right]$

$Z_{3}^{1} \leq 0$

$Z_{4}=Q-Q^{*}-Q^{*} \operatorname{Ln} \frac{Q}{Q^{*}}$

$Z_{4}^{1}=-\left(\frac{Q^{*}-Q}{Q}\right)[v I(v+\mu+\delta) Q]$

$Z_{4}^{1}=-\left(Q^{*}-Q\right)\left[v \frac{I^{*}}{Q^{*}}(v+\mu+\delta)\right]$

For equilibrium position

$\gamma I^{*}=(v+\mu+\delta) Q^{*}$

$\frac{I^{*}}{Q^{*}}=\frac{=(v+\mu+\delta)}{v}$

$Z_{4}^{1}=-\left(Q^{*}-Q\right)\left[\frac{v(v+\mu+\delta)}{v^{*}}-(v+\mu+\delta)\right]$

$Z_{4}^{1} \leq 0$

$Z_{5}=R-R^{*}-R^{*} \operatorname{Ln} \frac{R}{R^{*}}$

$Z_{5}^{1}=-\left(\frac{R^{*}-R}{R}\right)[v Q+\alpha I-(\mu+\rho) R]$

$Z_{5}^{1} \leq-\left(R^{*}-R\right)\left[\frac{v Q^{*}+\alpha I^{*}}{R^{*}}(\mu+\rho)\right]$
For equilibrium position

$v Q^{*}+\alpha I^{*}=(\mu+\rho) R^{*}$

$\frac{v Q^{*}+\alpha I^{*}}{R^{*}}=(\mu+\rho)$

$Z_{5}^{1} \leq-\left(R^{*}-R\right)[(\mu+\rho)-(\mu+\rho)]$

$Z_{5}^{1} \leq 0$

$Z_{6}=V_{1}-V_{1}^{*}-V_{1}^{*} \operatorname{Ln} \frac{V_{1}}{V_{1}^{*}}$

$Z_{6}^{1}=-\left(\frac{V_{1}^{*}-V_{1}}{V_{1}}\right)\left[\left(\sigma_{1_{-}}+\varepsilon_{1}+\mu\right) V_{1}\right]$

$Z_{6}^{1} \leq-\left(V_{1}^{*}-V_{1}\right)\left[\frac{\sigma_{1} S^{*}+\alpha I^{*}}{V_{1}^{*}}-\left(\sigma_{1-}+\varepsilon_{1}+\mu\right)\right]$

For equilibrium position

$\sigma_{1_{-}} S^{*}=\left(\sigma_{2_{-}}+\varepsilon_{1}+\mu\right) V_{1}^{*}$

$\frac{\sigma_{1} S^{*}}{V_{1}^{*}}=\left(\sigma_{2_{-}}+\varepsilon_{1}+\mu\right)$

$Z_{6}^{1} \leq-\left(V_{1}^{*}-V_{1}\right)\left[\left(\sigma_{2_{-}}+\varepsilon_{1}+\mu\right)-\left(\sigma_{2_{-}}+\varepsilon_{1}+\mu\right)\right]$

$Z_{6}^{1} \leq 0$

$Z_{7}=V_{2}-V_{2}^{*}-V_{2}^{*} \operatorname{Ln} \frac{V_{2}}{V_{2}^{*}}$

$Z_{7}^{1}=-\left(\frac{V_{2}^{*}-V_{2}}{V_{2}}\right)\left[\sigma_{2-} V_{1}-\left(\varepsilon_{2}+\mu\right) V_{2}\right]$

$Z_{7}^{1} \leq-\left(V_{1}^{*}-V_{1}\right)\left[\frac{\sigma_{2}-V_{1}^{*}}{V_{2}^{*}}-\left(\varepsilon_{2}+\mu\right)\right]$

For equilibrium position

$\sigma_{2} V_{1}^{*}=\left(\varepsilon_{2}+\mu\right) V_{2}^{*}$

$\frac{\sigma_{2} V_{1}^{*}}{V_{2}^{*}}=\varepsilon_{2}+\mu$

$Z_{7}^{1} \leq-\left(V_{2}^{*}-V_{2}\right)\left[\left(\varepsilon_{2}+\mu\right)-\left(\varepsilon_{2}+\mu\right)\right]$

$Z_{7}^{1} \leq 0$

Therefore, $\mathrm{Z}$ defines:

$\mathrm{Z}=\sum_{i=1}^{n} Z_{i}=\sum_{i=1}^{n} C_{i}\left(\chi_{i}-\chi_{i}^{*}-\chi_{i}^{*} \ln \frac{\chi_{i}}{\chi_{i}^{*}}\right.$

$\mathrm{Z}=Z_{1}+Z_{2}+Z_{3}+Z_{4}+Z_{5}+Z_{6}+Z_{7}$

Thus $Z^{1}=\sum_{i=1}^{n} Z_{i}^{1}$

$Z^{1} \leq 0$ for $R_{0}^{h}>1$.

In a Lyapunov function for system (1), arbitrary constant $C_{i}$ can be chosen from $\mathrm{R}_{+}^{7}$ and any linear combination of $\mathrm{Z}$ would be a lyapunov function for the function for the system. In $\mathrm{D}$ and it follows by Lesolle's Invariance Principle [16]. That every solution to the equations of the model approaches the associated unique endemic equilibrium ( $\mathrm{P}^{*}$ ), of the model as $\mathrm{t} \rightarrow \infty$ for $\mathrm{R}_{0}{ }^{\mathrm{h}}>1$.

Numerical simulation

Numerical parameter values 


\begin{tabular}{|c|c|c|}
\hline Parameter & value & Source \\
\hline$\beta$ & 0.0000245 & calculated \\
\hline$\eta$ & 0.1 & 1 \\
\hline$\gamma$ & 0.0998 & 10 \\
\hline a & 0.125 & 2 \\
\hline v & 0.125 & 2 \\
\hline$\sigma 1$ & 0.915 & 8 \\
\hline$\sigma 2$ & 0.921 & 9 \\
\hline$\varepsilon 1$ & 0.001 & 3 \\
\hline$\varepsilon 2$ & 0 & 3 \\
\hline$\rho$ & 0 & 4 \\
\hline$\delta$ & 0.0000012 & 7 \\
\hline$\mu \mathrm{h}$ & 0.00002192 & 11 \\
\hline $\mathrm{Bh}$ & 0.00003397 & 11 \\
\hline
\end{tabular}

Table 2: Measles simulation parameter values.

\section{Results and Discussion}
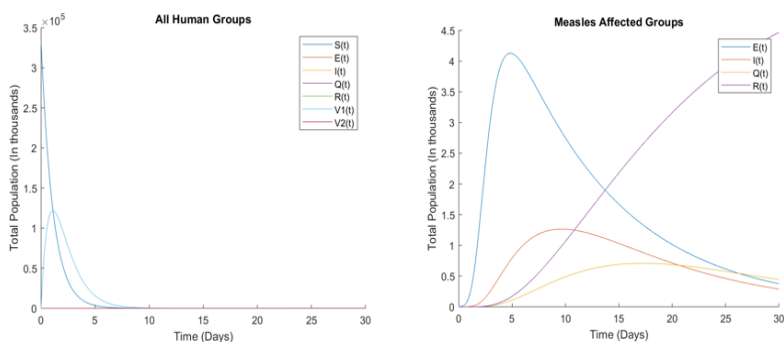

Figure 2a and 2b: Analysis of measles in the United States population.

In the first set of simulations we are analyzing the actual United States measles epidemic with the parameters given in table 2. Figure 2a includes all of the groups, however, the susceptible and vaccinated groups overshadow all of the others. So, in figure $2 b$ we focused only on the groups that came into contact with the measles virus. We see that the peak of the infectious population occurs around nine to ten days while the peak exposed population occurs around four to five days. This gives our simulation an incubation period of approximately four to six days, which is slightly less than the actual incubation period at 8 - 12 days [7]. The recovered group
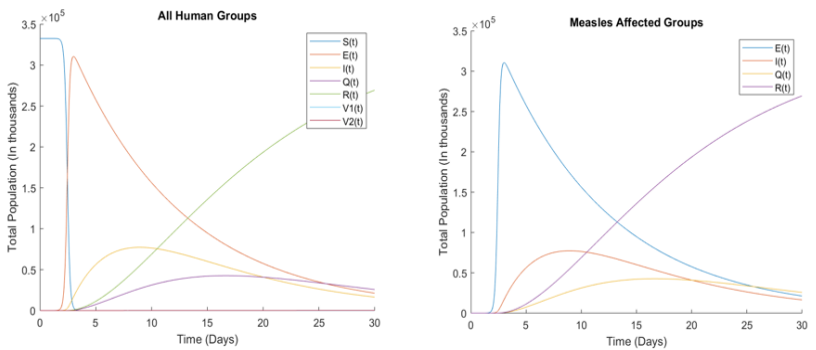

Figure 3a and 3b: Analysis of measles in the unvaccinated United States population. continues to grow past the simulated month, beginning to plateau out towards the end. Showing that the recovered group only grows and never shrinks represents the life lasting immunity to measles after the infection subsides [4].

For figures 3 we used the same graphs as we did for figure two as a means of comparison. We see that vaccinations had a substantial effect on the spread of measles. With figure $2 b$ we only saw about 1.26 thousand people infected with measles and over 4.5 thousand recovered. Figures 3 show that the infectious population would have reached up to 7.725 million people at its peak if no one had received the MMR vaccine. The comparison between figures 2 and 3 show just how important the role of vaccination is when dealing with a highly infectious disease. Vaccinations had resulted in over a $613,095 \%$ decrease in infectious measles cases.

\section{Conclusion}

We had developed a SEIQRV $V_{1} \mathrm{~V}_{2}$ model in order to analyze the 2019 measles spread in the United States. Using CDC data and viral data, we proved that our measles simulation followed the same time frames of the actual virus and similar case numbers.

With the two examples shown, we found that vaccinations were extremely important in limiting the spread of the virus. In the actual population only 1,260 people were infected at the disease's peak, but with the unvaccinated population we saw that number rise to 7.725 million. Such low numbers in the actual population are backed by the basic reproduction number coming out to 0.03976 which is less than 1 . This number and the graphs both show that measles has a difficult time spreading when most people are vaccinated against the virus.

\section{Competing Interests}

The authors declare no competing interests.

\section{Data Availability}

Code Availability

The MATLAB code used for the simulated graphs can be obtained upon request to the corresponding author.

\section{Author Contributions}

Designed the project (D. S., N. K., S.P.), performed simulations (D. S., N. K.), performed analysis of the data (D. S., N. K.), wrote the paper (D. S., N. K., S.P.).

\section{Bibliography}

1. MacFadden DR and Gold WL. "Measles". CMAJ: Canadian Medical Association Journal 186.6 (2014): 450.

2. White SJ., et al. "Measles, mumps, and rubella". Clinical Obstetrics and Gynecology 55.2 (2012): 550-559. 
3. La Torre G., et al. "The effectiveness of measles-mumps-rubella (MMR) vaccination in the prevention of pediatric hospitalizations for targeted and untargeted infections: A retrospective cohort study". Human Vaccines and Immunotherapeutics 13.8 (2017): 1879-1883.

4. Griffin DE. "The Immune Response in Measles: Virus Control, Clearance and Protective Immunity". Viruses 8.10 (2016): 282.

5. Patel M., et al. "National Update on Measles Cases and Outbreaks - United States, January 1-October 1, 2019". Morbidity and Mortality Weekly Report 68 (2019): 893-896.

6. Patel M., et al. "Increase in Measles Cases - United States, January 1-April 26, 2019". Morbidity and Mortality Weekly Report 68 (2019): 402-404.

7. Pickering L., et al. "Red Book: 2009 Report of the Committee on Infectious Diseases (28 ${ }^{\text {th }}$ edition)". American Academy of Pediatrics (2009).

8. CDC/National Center for Health Statistics. "Vaccination coverage for selected diseases among children aged 19-35 months, by race, Hispanic origin, poverty level, and location of residence in metropolitan statistical area: United States, selected years 1998-2017" (2017).

9. CDC/National Center for Health Statistics. "Vaccination coverage for selected diseases among adolescents aged 13-17 years, by selected characteristics: United States, selected years 2008-2017" (2017).

10. National Notifiable Diseases Surveillance System (NNDSS). "Measles Cases and Outbreaks" (2020).

11. Central Intelligence Agency. "The World Factbook: United States" (2018).

12. Kamgang JC and Sallet G. "Computation of threshold conditions for epidemiological models and global stability of the disease-free equilibrium (DFE)". Mathematical Biosciences 213.1 (2008): 1-12.

13. Shuai $\mathrm{Z}$ and van den Driessche P. "Global Stability of Infectious Disease Models Using Lyapunov Functions". SIAM Journal on Applied Mathematics 73 (2013): 1513-1532.

14. Safi MA and Garba SM. "Global Stability Analysis of SEIR Model with Holling Type II Incidence Function". Computational and Mathematical Methods in Medicine (2012): 826052.

15. Melesse DY and Gumel AB. "Global asymptotic properties of an SEIRS model with multiple infectious stages". Journal of Mathematical Analysis and Applications 366.1 (2010): 202217.
16. Li MY., et al. "Global Dynamics of an SEIR Epidemic Model with Vertical Transmission". SIAM Journal on Applied Mathematics 62.1 (2001): 58-69.

\section{Assets from publication with us}

- Prompt Acknowledgement after receiving the article

- Thorough Double blinded peer review

- Rapid Publication

- Issue of Publication Certificate

- High visibility of your Published work

Website: $w w w$.actascientific.com/

Submit Article: www.actascientific.com/submission.php Email us: editor@actascientific.com

Contact us: +919182824667 